\title{
Simple algebraic solutions to the kinetic problems of triangle, quadrangle and pentangle reactions
}

\author{
Roland Tóbiás $^{1} \cdot$ Gyula Tasi $^{1}$
}

Received: 14 June 2015 / Accepted: 16 August 2015 / Published online: 26 August 2015

(C) Springer International Publishing Switzerland 2015

\begin{abstract}
In this paper, ignoring graph-theoretic tools, simple algebraic solutions are presented for the kinetic problems of the triangle, quadrangle and pentangle reactions with the help of Krylov and Vandermonde matrices. As far as we know at the time of writing, this is the first time for the cases of the quadrangle and pentangle reactions. Our solutions do not require diagonalizability and non-degenerate eigenvalues concerning the coefficient matrix of the first-order reaction network.
\end{abstract}

Keywords First-order reaction network - Algebraic model $\cdot$ Krylov and Vandermonde matrices · Triangle · Quadrangle and pentangle reactions · Analytical and numerical solutions

\section{Introduction}

Numerous papers have been devoted to the study of mathematical modeling of firstorder reaction networks in the literature. It is worth mentioning the work of Wei and Prater [1], which, assuming that the so-called kinetic matrix can be diagonalized and its eigenvalues are non-degenerate, has presented a general solution based on matrix algebra. Further studies, applying classical integration technique [2], matrix and convolution methods [3,4], special approximation [5] as well as Laplace transformation $[6,7]$, supplied analytical solutions for the kinetic problem of the triangle reaction. Eykholt has presented a general analytical solution for networks of irreversible firstorder reactions [8].

Gyula Tasi

tasi@chem.u-szeged.hu

1 Department of Applied and Environmental Chemistry, University of Szeged, Rerrich B. tér 1, Szeged 6720, Hungary 
Very recently, the flow-graph theory and the Cramer's rule have been used to obtain analytical solutions for the reaction systems under consideration [9-11]. Similarly, in the study of Karmakar and Mandal graph-theoretic reasoning was used, too [12].

In this paper, ignoring graph-theoretic tools, simple algebraic solutions are presented to the kinetic problems of triangle, quadrangle and pentangle reactions with the help of Krylov and Vandermonde matrices [13]. Our solutions do not require diagonalizability and non-degenerate eigenvalues concerning the coefficient matrix of the first-order reaction network.

\section{A simple algebraic model and its solution}

Let us consider a closed, homogeneous reaction system with $K$ chemical components $\left(A_{\mathrm{j}}, j=1,2, \ldots, K\right)$ and $R$ elementary chemical reactions $\left(r_{\mathrm{i}}, i=1,2, \ldots, R\right)$ at constant temperature, pressure and volume. The stoichiometric equations describing the stoichiometry of the chemical reactions under consideration are as follows:

$$
\sum_{j=1}^{K} v_{i j} A_{j}=0 \quad(i=1,2, \ldots, R)
$$

where $\left\{v_{i j}\right\}$ are the well-known stoichiometric coefficients of the components forming the stoichiometric matrix $\mathbf{S}$ of the reaction system $\left(\mathbf{S}=\left\{v_{i j}\right\}, \operatorname{dim}(\mathbf{S})=R \times K\right)$. The stoichiometric equations can also be written in a more detailed form:

$$
\sum_{j=1}^{K} d_{i j} A_{j}=\sum_{j=1}^{K} g_{i j} A_{j} \quad(i=1,2, \ldots, R)
$$

with the help of the consumption $\mathbf{D}=\left\{d_{i j}\right\}$ and formation $\mathbf{G}=\left\{g_{i j}\right\}$ matrices. The elements of the $\mathbf{D}$ and $\mathbf{G}$ matrices are nonnegative integers. The stoichiometric matrix $\mathbf{S}$ can then be expressed as in Eq. (3).

$$
\mathbf{S}=\mathbf{G}-\mathbf{D}
$$

The concentration vector $\mathbf{C}$ of the chemical components, $\mathbf{C}=\left\{c_{j}\right\}$, varies with time according to the following equations [14]:

$$
\dot{c}_{j}=\sum_{i=1}^{R} v_{i j} r_{i} \quad(j=1,2, \ldots, K)
$$

where $\dot{c}_{j}$ is the time derivative of the concentration of the component $A_{j}$, and $r_{i}$ is the reaction rate of the $i$ th elementary chemical reaction $[15,16]$. Equation (4) can also be written in matrix form: 


$$
\dot{\mathbf{C}}=\mathbf{S}^{\mathrm{T}} \cdot \rho
$$

where $\dot{\mathbf{C}}=\left\{\dot{c}_{j}\right\}, \boldsymbol{\rho}=\left\{r_{i}\right\}$, and $\mathbf{S}^{\mathrm{T}}$ is the transpose of the stoichiometric matrix.

If the concentrations are not too large, the reaction rates can be computed according to the law of mass action $[17,18]$ :

$$
r_{i}=k_{i} \prod_{j=1}^{K} c_{j}^{d_{i j}} \quad(i=1,2, \ldots, R)
$$

where $k_{i}$ is the reaction rate coefficient of the $i$ th elementary chemical reaction. The value of $k_{i}$ depends only on the temperature and, occasionally, on the pressure as well as some other parameters.

Since the derivatives $\left\{\dot{c}_{j}\right\}$ are firs-order homogeneous functions of the concentrations $\left\{c_{j}\right\}$ for a first-order reaction network, Euler's theorem holds for them:

$$
\dot{c}_{j}=\sum_{m=1}^{K} f_{j m} c_{m} \quad(j=1,2, \ldots, K)
$$

where $f_{j m}=\partial \dot{c}_{j} / \partial c_{m}$. Equation(7) can be written in matrix form as follows:

$$
\dot{\mathbf{C}}=\mathbf{F C}
$$

where $\mathbf{F}=\partial \dot{\mathbf{C}} / \partial \mathbf{C}=\left\{f_{j m}\right\}$ is the Jacobian, which does not depend on time. It is called the coefficient matrix of the first-order reaction network.

Equation (7) is a system of first-order, homogeneous, linear differential equations with initial condition $\mathbf{C}_{0}=\mathbf{C}(0)$. Its solution can be taken in the following form [13]:

$$
\mathbf{C}(t)=\mathbf{F}_{\mathbf{C}_{0}} \mathbf{V}^{-1} \mathbf{E}(t)
$$

where

$$
\begin{aligned}
\mathbf{F}_{\mathbf{C}_{0}}= & \left\{\mathbf{C}_{0}, \mathbf{F C}_{0}, \mathbf{F}^{2} \mathbf{C}_{0}, \ldots, \mathbf{F}^{K-1} \mathbf{C}_{0}\right\} \\
\mathbf{E}(t)= & \left\{e^{\lambda_{1} t}, t e^{\lambda_{1} t}, t^{2} e^{\lambda_{1} t} \ldots, t^{\mu_{1}-1} e^{\lambda_{1} t}, \ldots, e^{\lambda_{L} t},\right. \\
& \left.t e^{\lambda_{L} t}, t^{2} e^{\lambda_{L} t} \ldots, t^{\mu_{L}-1} e^{\lambda_{L} t}\right\}^{T} \\
\mathbf{V}= & \left\{\mathbf{E}(0), \dot{\mathbf{E}}(0), \ddot{\mathbf{E}}(0), \ldots, \mathbf{E}^{(K-1)}(0)\right\}
\end{aligned}
$$

$\mathbf{F}_{\mathbf{C}_{0}}, \mathbf{E}(\mathrm{t})$ and $\mathbf{V}$ in Eqs. (10)-(12) are the Krylov matrix, the time propagation vector and the Vandermonde matrix, respectively. The parameter $L$ in Eq. (11) is the number of distinct eigenvalues of matrix $\mathbf{F}, \lambda_{k}$ is the $k$ th eigenvalue, and $\mu_{k}$ is its multiplicity. The columns of matrix $\mathbf{V}$ in Eq. (12) consist of various derivatives of vector $\mathbf{E}(\mathrm{t})$ taken at $\mathrm{t}=0$. Matrix $\mathbf{V}$ is regular, so it has inverse. 
It can be shown that $\sum_{j=1}^{K} \dot{c}_{j}=0$ fulfills at any instant of time, so the matrix $\mathbf{F}$ is singular. In that case, we can assume that $\lambda_{L}=0$. Furthermore, if $\mu_{L} \neq 1$, then divergence occurs, therefore, $\mu_{L}=1$.

Since $\lambda_{L}=0$, we should locate the remaining $(L-1)$ eigenvalues. This can be accomplished in closed form if $L-1 \leq 4$, i.e., $L \leq 5$. Because we also have the relation $L \leq K$, we can therefore determine the eigenvalues algebraically only for $K \leq 5$. To determine the eigenvalues of matrix $\mathbf{F}$, we need to know its characteristic polynomial. The coefficients of the characteristic polynomial can be obtained, for instance, with the help of Leverrier's method [19,20]:

$$
\begin{aligned}
a_{K+1} & =(-1)^{K} \\
a_{K+1-n} & =-\frac{1}{n} \sum_{i=1}^{n} a_{K+1-n+i} \operatorname{tr}\left(\mathbf{F}^{i}\right) \quad(n=1,2, \ldots, K)
\end{aligned}
$$

where $a_{l}$ is the coefficient of the term $\lambda^{l-1}$, and $\operatorname{tr}\left(\mathbf{F}^{i}\right)$ is the trace of the power matrix $\mathbf{F}^{i}$. Since $a_{1}=0(\operatorname{det}(\mathbf{F})=0)$, we should solve a $(K-1)$ th degree polynomial equation.

\section{An illustration: the triangle reaction}

For illustration, the simplest case, the kinetic problem of the triangle reaction, is solved. Its analytical solution has already been published in the literature [10,12]. The kinetic model of the triangle reaction can be seen in Fig. 1 .

According to Eq. (4), the time derivatives of the concentrations of the components are as follows:

$$
\begin{aligned}
& \dot{c}_{1}=-\left(k_{1}+k_{6}\right) c_{1}+k_{2} c_{2}+k_{5} c_{3} \\
& \dot{c}_{2}=k_{1} c_{1}-\left(k_{2}+k_{3}\right) c_{2}+k_{4} c_{3} \\
& \dot{c}_{3}=k_{6} c_{1}+k_{3} c_{2}-\left(k_{4}+k_{5}\right) c_{3} .
\end{aligned}
$$

Fig. 1 The kinetic model of the triangle reaction

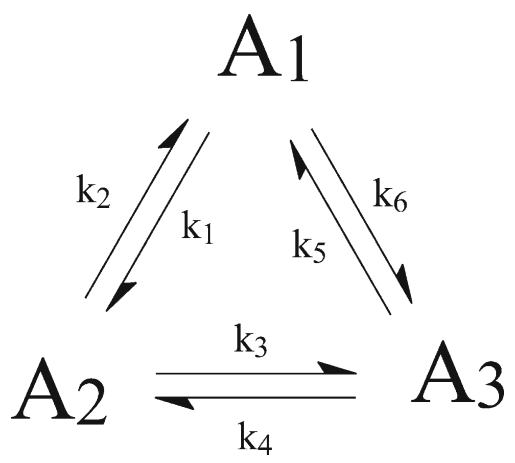


The $\mathbf{F}$ matrix of the reaction system in question comes from Eq. (8):

$$
\mathbf{F}=\left(\begin{array}{ccc}
-\left(k_{1}+k_{6}\right) & k_{2} & k_{5} \\
k_{1} & -\left(k_{2}+k_{3}\right) & k_{4} \\
k_{6} & k_{3} & -\left(k_{4}+k_{5}\right)
\end{array}\right)
$$

The coefficients of the characteristic polynomial of $\mathbf{F}$ in Eq. (15) can be obtained via Eq. (13):

$$
\begin{aligned}
& a_{4}=(-1)^{3}=-1, \\
& a_{3}=-a_{4} \operatorname{tr}(\mathbf{F}), \\
& a_{2}=-\frac{1}{2}\left(a_{3} \operatorname{tr}(\mathbf{F})+a_{4} \operatorname{tr}\left(\mathbf{F}^{2}\right)\right) \\
& a_{1}=0
\end{aligned}
$$

where

$$
\begin{aligned}
\operatorname{tr}(\mathbf{F})= & \sum_{i=1}^{3} f_{i i}=-\sum_{l=1}^{6} k_{l} \\
\operatorname{tr}\left(\mathbf{F}^{2}\right)= & \left(k_{1}+k_{6}\right)^{2}+\left(k_{2}+k_{3}\right)^{2} \\
& +\left(k_{4}+k_{5}\right)^{2}+2 k_{1} k_{2}+2 k_{3} k_{4}+2 k_{5} k_{6}
\end{aligned}
$$

In order to get the eigenvalues of matrix $\mathbf{F}$, we should solve the following cubic equation:

$$
a_{4} \lambda^{3}+a_{3} \lambda^{2}+a_{2} \lambda=0
$$

However, $\lambda_{3}=0$, therefore, dividing Eq. (18) by $\lambda$, a quadratic equation is obtained:

$$
a_{4} \lambda^{2}+a_{3} \lambda+a_{2}=0
$$

which can easily be solved.

First, let us assume that the eigenvalues $\left(\lambda_{1}, \lambda_{2}\right)$ are distinct. In this case

$$
\mathbf{E}(t)=\left\{e^{\lambda_{1} t}, e^{\lambda_{2} t}, 1\right\}^{T}
$$

With the help of Eq. (20), the columns of matrix $\mathbf{V}$ can be determined according to Eq. (12):

$$
\begin{aligned}
& \mathbf{E}(0)=\left\{e^{\lambda_{1} t}, e^{\lambda_{2} t}, 1\right\}_{t=0}^{T}=\{1,1,1\}^{T} \\
& \dot{\mathbf{E}}(0)=\left\{\lambda_{1} e^{\lambda_{1} t}, \lambda_{2} e^{\lambda_{2} t}, 0\right\}_{t=0}^{T}=\left\{\lambda_{1}, \lambda_{2}, 0\right\}^{T} \\
& \ddot{\mathbf{E}}(0)=\left\{\lambda_{1}^{2} e^{\lambda_{1} t}, \lambda_{2}^{2} e^{\lambda_{2} t}, 0\right\}_{t=0}^{T}=\left\{\lambda_{1}^{2}, \lambda_{2}^{2}, 0\right\}^{T}
\end{aligned}
$$


After all, matrix $\mathbf{V}$ is as follows:

$$
\mathbf{V}=\left(\begin{array}{ccc}
1 & \lambda_{1} & \lambda_{1}^{2} \\
1 & \lambda_{2} & \lambda_{2}^{2} \\
1 & 0 & 0
\end{array}\right)
$$

The inverse of matrix $\mathbf{V}$ in Eq. (22):

$$
\mathbf{V}^{-1}=\left(\begin{array}{ccc}
0 & 0 & 1 \\
-\frac{\lambda_{2}}{\lambda_{1}\left(\lambda_{1}-\lambda_{2}\right)} & -\frac{\lambda_{1}}{\lambda_{2}\left(\lambda_{2}-\lambda_{1}\right)} & -\frac{\lambda_{1}+\lambda_{2}}{\lambda_{1} \lambda_{2}} \\
\frac{1}{\lambda_{1}\left(\lambda_{1}-\lambda_{2}\right)} & \frac{1}{\lambda_{2}\left(\lambda_{2}-\lambda_{1}\right)} & \frac{1}{\lambda_{1} \lambda_{2}}
\end{array}\right)
$$

The next task is the determination of the elements of matrix $\mathbf{F}_{\mathbf{C}_{0}}$ :

$$
\begin{aligned}
\left(\mathbf{F}_{\mathbf{C}_{0}}\right)_{11}= & c_{01} \\
\left(\mathbf{F}_{\mathbf{C}_{0}}\right)_{21}= & c_{02} \\
\left(\mathbf{F}_{\mathbf{C}_{0}}\right)_{31}= & c_{03} \\
\left(\mathbf{F}_{\mathbf{C}_{0}}\right)_{12}= & -\left(k_{1}+k_{6}\right) c_{01}+k_{2} c_{02}+k_{5} c_{03} \\
\left(\mathbf{F}_{\mathbf{C}_{0}}\right)_{22}= & k_{1} c_{01}-\left(k_{2}+k_{3}\right) c_{02}+k_{4} c_{03} \\
\left(\mathbf{F}_{\mathbf{C}_{0}}\right)_{32}= & k_{6} c_{01}+k_{3} c_{02}-\left(k_{4}+k_{5}\right) c_{03} \\
\left(\mathbf{F}_{\mathbf{C}_{0}}\right)_{13}= & {\left[\left(k_{1}+k_{6}\right)^{2}+k_{1} k_{2}+k_{5} k_{6}\right] c_{01}+\left[k_{3} k_{5}-k_{2}\left(k_{1}+k_{2}+k_{3}+k_{6}\right)\right] c_{02} } \\
& +\left[k_{2} k_{4}-k_{5}\left(k_{1}+k_{4}+k_{5}+k_{6}\right)\right] c_{03} \\
\left(\mathbf{F}_{\mathbf{C}_{0}}\right)_{23}= & {\left[k_{4} k_{6}-k_{1}\left(k_{1}+k_{2}+k_{3}+k_{6}\right)\right] c_{01}+\left[\left(k_{2}+k_{3}\right)^{2}+k_{1} k_{2}+k_{3} k_{4}\right] c_{02} } \\
& +\left[k_{1} k_{5}-k_{4}\left(k_{2}+k_{3}+k_{4}+k_{5}\right)\right] c_{03} \\
\left(\mathbf{F}_{\mathbf{C}_{0}}\right)_{33}= & {\left[k_{1} k_{3}-k_{6}\left(k_{1}+k_{4}+k_{5}+k_{6}\right)\right] c_{01}+\left[k_{2} k_{6}-k_{3}\left(k_{2}+k_{3}+k_{4}+k_{5}\right)\right] c_{02} } \\
& +\left[\left(k_{4}+k_{5}\right)^{2}+k_{3} k_{4}+k_{5} k_{6}\right] c_{03}
\end{aligned}
$$

The concentrations of the components can now be obtained analytically according to Eq. (9):

$$
c_{j}=\sum_{k=1}^{3} \sum_{l=1}^{3}\left(\mathbf{F}_{\mathbf{C}_{0}}\right)_{j k}\left(\mathbf{V}^{-1}\right)_{k l} e^{\lambda_{l} t} \quad(j=1,2,3)
$$

If $\lambda_{1}=\lambda_{2}=\eta$, which case is generally not treated in the literature $[10,12]$, the time propagation vector $\mathbf{E}$ and its derivatives are the followings:

$$
\begin{aligned}
& \mathbf{E}(0)=\left\{e^{\eta t}, t e^{\eta t}, 1\right\}_{t=0}^{T}=\{1,0,1\}^{T} \\
& \dot{\mathbf{E}}(0)=\left\{\eta e^{\eta t}, e^{\eta t}+\eta t e^{\eta t}, 0\right\}_{t=0}^{T}=\{\eta, 1,0\}^{T} \\
& \ddot{\mathbf{E}}(0)=\left\{\eta^{2} e^{\eta t}, 2 \eta e^{\eta t}+\eta^{2} t e^{\eta t}, 0\right\}_{t=0}^{T}=\left\{\eta^{2}, 2 \eta, 0\right\}^{T}
\end{aligned}
$$


With the help of Eq. (26), matrix $\mathbf{V}$ and its inverse $\mathbf{V}^{-1}$ can easily be constructed:

$$
\begin{aligned}
\mathbf{V} & =\left(\begin{array}{ccc}
1 & \eta & \eta^{2} \\
0 & 1 & 2 \eta \\
1 & 0 & 0
\end{array}\right) \\
\mathbf{V}^{-1} & =\left(\begin{array}{ccc}
0 & 0 & 1 \\
2 / \eta & -1 & -2 / \eta \\
-1 / \eta^{2} & 1 / \eta & 1 / \eta^{2}
\end{array}\right)
\end{aligned}
$$

The elements of matrix $\mathbf{F}_{\mathbf{C}_{0}}$ are the same, therefore the concentrations of the components are as follows:

$$
c_{j}=\sum_{k=1}^{3} \sum_{l=1}^{3}\left(\mathbf{F}_{\mathbf{C}_{0}}\right)_{j k}\left(\mathbf{V}^{-1}\right)_{k l} \varepsilon_{l}(t) \quad(j=1,2,3)
$$

where $\varepsilon_{l}(t)$ is the $l$ th component of vector $\mathbf{E}(\mathrm{t})$.

\section{Analytical solution of the kinetic problem of quadrangle reaction}

In what follows, we present, for the first time, a simple analytical solution to the kinetic problem of the quadrangle reaction $(K=4)$. Figure 2 shows the kinetic model of the reaction system.

The differential balance equations for the chemical components are as follows:

$$
\begin{aligned}
& \dot{c}_{1}=-\left(k_{1}+k_{8}+k_{9}\right) c_{1}+k_{2} c_{2}+k_{10} c_{3}+k_{7} c_{4} \\
& \dot{c}_{2}=k_{1} c_{1}-\left(k_{2}+k_{3}+k_{12}\right) c_{2}+k_{4} c_{3}+k_{11} c_{4} \\
& \dot{c}_{3}=k_{9} c_{1}+k_{3} c_{2}-\left(k_{4}+k_{6}+k_{10}\right) c_{3}+k_{5} c_{4} \\
& \dot{c}_{4}=k_{8} c_{1}+k_{12} c_{2}+k_{6} c_{3}-\left(k_{5}+k_{7}+k_{11}\right) c_{4}
\end{aligned}
$$

Fig. 2 The kinetic model of the quadrangle reaction

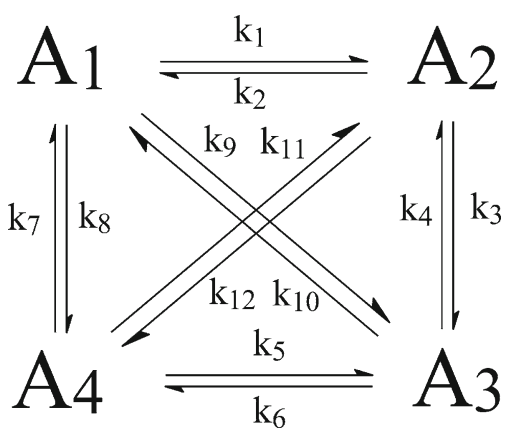


The coefficient matrix of the system of equations in Eq. (29) can be seen in Eq. (30).

$$
\mathbf{F}=\left(\begin{array}{cccc}
-\left(k_{1}+k_{8}+k_{9}\right) & k_{2} & k_{10} & k_{7} \\
k_{1} & -\left(k_{2}+k_{3}+k_{12}\right) & k_{4} & k_{11} \\
k_{9} & k_{3} & -\left(k_{4}+k_{6}+k_{10}\right) & k_{5} \\
k_{8} & k_{12} & k_{6} & -\left(k_{5}+k_{7}+k_{11}\right)
\end{array}\right)
$$

The following expressions can be obtained for the coefficients of the characteristic polynomial of matrix $\mathbf{F}$ in Eq. (30):

$$
\begin{aligned}
& a_{5}=(-1)^{4}=1 \\
& a_{4}=-a_{5} \operatorname{tr}(\mathbf{F}) \\
& a_{3}=-\frac{1}{2}\left(a_{4} \operatorname{tr}(\mathbf{F})+a_{5} \operatorname{tr}\left(\mathbf{F}^{2}\right)\right) \\
& a_{2}=-\frac{1}{3}\left(a_{3} \operatorname{tr}(\mathbf{F})+a_{4} \operatorname{tr}\left(\mathbf{F}^{2}\right)+a_{5} \operatorname{tr}\left(\mathbf{F}^{3}\right)\right) \\
& a_{1}=0
\end{aligned}
$$

where

$$
\begin{aligned}
\operatorname{tr}(\mathbf{F}) & =\sum_{i=1}^{4} f_{i i} \\
\operatorname{tr}\left(\mathbf{F}^{2}\right) & =\sum_{i=1}^{4} \sum_{j=1}^{4} f_{i j} f_{j i} \\
\operatorname{tr}\left(\mathbf{F}^{3}\right) & =\sum_{i=1}^{4} \sum_{j=1}^{4}\left(\mathbf{F}^{2}\right)_{i j} f_{j i}=\sum_{i=1}^{4} \sum_{j=1}^{4} \sum_{k=1}^{4} f_{i k} f_{k j} f_{j i}
\end{aligned}
$$

According to Eqs. (31)-(34), the 4th degree characteristic polynomial equation is the following:

$$
a_{5} \lambda^{4}+a_{4} \lambda^{3}+a_{3} \lambda^{2}+a_{2} \lambda=0
$$

We know that $\lambda_{4}=0$, and dividing Eq. (35) by $\lambda$, we get a cubic equation:

$$
a_{5} \lambda^{3}+a_{4} \lambda^{2}+a_{3} \lambda+a_{2}=0
$$

which can be solved with the help of Cardano's formula [21].

Let us assume first that the eigenvalues are all distinct. In this case

$$
\mathbf{E}(t)=\left\{e^{\lambda_{1} t}, e^{\lambda_{2} t}, e^{\lambda_{3} t}, 1\right\}^{T}
$$


With the help of Eq. (37), the columns of matrix $\mathbf{V}$ can be constructed according to Eq. (12):

$$
\begin{aligned}
& \mathbf{E}(0)=\left\{e^{\lambda_{1} t}, e^{\lambda_{2} t}, e^{\lambda_{3} t}, 1\right\}_{t=0}^{T}=\{1,1,1,1\}^{T} \\
& \dot{\mathbf{E}}(0)=\left\{\lambda_{1} e^{\lambda_{1} t}, \lambda_{2} e^{\lambda_{2} t}, \lambda_{3} e^{\lambda_{3} t}, 0\right\}_{t=0}^{T}=\left\{\lambda_{1}, \lambda_{2}, \lambda_{3}, 0\right\}^{T} \\
& \ddot{\mathbf{E}}(0)=\left\{\lambda_{1}^{2} e^{\lambda_{1} t}, \lambda_{2}^{2} e^{\lambda_{2} t}, \lambda_{3}^{2} e^{\lambda_{3} t}, 0\right\}_{t=0}^{T}=\left\{\lambda_{1}^{2}, \lambda_{2}^{2}, \lambda_{3}^{2}, 0\right\}^{T} \\
& \ddot{\mathbf{E}}(0)=\left\{\lambda_{1}^{3} e^{\lambda_{1} t}, \lambda_{2}^{3} e^{\lambda_{2} t}, \lambda_{3}^{3} e^{\lambda_{3} t}, 0\right\}_{t=0}^{T}=\left\{\lambda_{1}^{3}, \lambda_{2}^{3}, \lambda_{3}^{3}, 0\right\}^{T}
\end{aligned}
$$

Equation (38) results in the following $\mathbf{V}$ matrix:

$$
\mathbf{V}=\left(\begin{array}{cccc}
1 & \lambda_{1} & \lambda_{1}^{2} & \lambda_{1}^{3} \\
1 & \lambda_{2} & \lambda_{2}^{2} & \lambda_{2}^{3} \\
1 & \lambda_{3} & \lambda_{3}^{2} & \lambda_{3}^{3} \\
1 & 0 & 0 & 0
\end{array}\right)
$$

The inverse of matrix $\mathbf{V}$ in Eq. (39) can easily be determined:

$$
\mathbf{V}^{-1}=\left(\begin{array}{cccc}
0 & 0 & 0 & 1 \\
\frac{\lambda_{2} \lambda_{3}}{\lambda_{1}\left(\lambda_{1}-\lambda_{2}\right)\left(\lambda_{1}-\lambda_{3}\right)} & \frac{\lambda_{1} \lambda_{3}}{\lambda_{2}\left(\lambda_{2}-\lambda_{1}\right)\left(\lambda_{2}-\lambda_{3}\right)} & \frac{\lambda_{1} \lambda_{2}}{\lambda_{3}\left(\lambda_{3}-\lambda_{1}\right)\left(\lambda_{3}-\lambda_{2}\right)} & -\frac{1}{\lambda_{1}}-\frac{\lambda_{2}+\lambda_{3}}{\lambda_{2} \lambda_{3}} \\
-\frac{\lambda_{2}+\lambda_{3}}{\lambda_{1}\left(\lambda_{1}-\lambda_{2}\right)\left(\lambda_{1}-\lambda_{3}\right)} & -\frac{\lambda_{1}+\lambda_{3}}{\lambda_{2}\left(\lambda_{2}-\lambda_{1}\right)\left(\lambda_{2}-\lambda_{3}\right)} & -\frac{\lambda_{1}+\lambda_{2}}{\lambda_{3}\left(\lambda_{3}-\lambda_{1}\right)\left(\lambda_{3}-\lambda_{2}\right)} & \frac{\lambda_{1}+\lambda_{2}+\lambda_{3}}{\lambda_{1} \lambda_{2} \lambda_{3}} \\
\frac{1}{\lambda_{1}\left(\lambda_{1}-\lambda_{2}\right)\left(\lambda_{1}-\lambda_{3}\right)} & \frac{1}{\lambda_{2}\left(\lambda_{2}-\lambda_{1}\right)\left(\lambda_{2}-\lambda_{3}\right)} & \frac{1}{\lambda_{3}\left(\lambda_{3}-\lambda_{1}\right)\left(\lambda_{3}-\lambda_{2}\right)} & -\frac{1}{\lambda_{1} \lambda_{2} \lambda_{3}}
\end{array}\right)
$$

Finally, only the elements of matrix $\mathbf{F}_{\mathbf{C}_{0}}$ should be generated:

$$
\begin{aligned}
& \left(\mathbf{F}_{\mathbf{C}_{0}}\right)_{p 1}=c_{0 p} \\
& \left(\mathbf{F}_{\mathbf{C}_{0}}\right)_{p 2}=\sum_{q=1}^{4} f_{p q} c_{0 q} \\
& \left(\mathbf{F}_{\mathbf{C}_{0}}\right)_{p 3}=\sum_{q=1}^{4} f_{p q}\left(\mathbf{F}_{\mathbf{C}_{0}}\right)_{q 2}=\sum_{q=1}^{4} \sum_{u=1}^{4} f_{p q} f_{q u} c_{0 u} \\
& \left(\mathbf{F}_{\mathbf{C}_{0}}\right)_{p 4}=\sum_{q=1}^{4} f_{p q}\left(\mathbf{F}_{\mathbf{C}_{0}}\right)_{q 3}=\sum_{q=1}^{4} \sum_{u=1}^{4} \sum_{v=1}^{4} f_{p q} f_{q u} f_{u v} c_{0 v}
\end{aligned}
$$

where $p=1,2,3,4$. The concentrations of the components can now be obtained analytically according to Eq. (9):

$$
c_{j}=\sum_{k=1}^{4} \sum_{l=1}^{4}\left(\mathbf{F}_{\mathbf{C}_{0}}\right)_{j k}\left(\mathbf{V}^{-1}\right)_{k l} e^{\lambda_{l} t} \quad(j=1,2,3,4)
$$


If there are degenerate eigenvalues, we can get the analytical solutions based on Eqs. (26)-(28).

\section{Analytical solution of the kinetic problem of pentangle reaction}

Finally, we present, for the first time, an analytical solution to the kinetic problem of the pentangle reaction $(K=5)$. Figure 3 shows the kinetic model of the reaction system.

The differential balance equations for the chemical components are as follows:

$$
\begin{aligned}
& \dot{c}_{1}=-\left(k_{1}+k_{10}+k_{15}+k_{19}\right) c_{1}+k_{2} c_{2}+k_{20} c_{3}+k_{16} c_{4}+k_{9} c_{5} \\
& \dot{c}_{2}=k_{1} c_{1}-\left(k_{2}+k_{3}+k_{14}+k_{18}\right) c_{2}+k_{4} c_{3}+k_{13} c_{4}+k_{17} c_{5} \\
& \dot{c}_{3}=k_{19} c_{1}+k_{3} c_{2}-\left(k_{4}+k_{5}+k_{12}+k_{20}\right) c_{3}+k_{6} c_{4}+k_{11} c_{5} \\
& \dot{c}_{4}=k_{15} c_{1}+k_{14} c_{2}+k_{5} c_{3}-\left(k_{6}+k_{7}+k_{13}+k_{16}\right) c_{4}+k_{8} c_{5} \\
& \dot{c}_{5}=k_{10} c_{1}+k_{18} c_{2}+k_{12} c_{3}+k_{7} c_{4}-\left(k_{8}+k_{9}+k_{11}+k_{17}\right) c_{5}
\end{aligned}
$$

The matrix F can easily be obtained based on Eq. (43):

$$
\mathbf{F}=\left(\begin{array}{ccccc}
-\left(\begin{array}{c}
k_{1}+k_{10}+ \\
k_{15}+k_{19}
\end{array}\right) & k_{2} & k_{20} & k_{16} & k_{9} \\
k_{1} & -\left(\begin{array}{c}
k_{2}+k_{3}+ \\
k_{14}+k_{18}
\end{array}\right) & k_{4} & k_{13} & k_{17} \\
k_{19} & k_{3} & -\left(\begin{array}{l}
k_{4}+k_{5}+ \\
k_{12}+k_{20}
\end{array}\right) & k_{6} & k_{11} \\
k_{15} & k_{14} & k_{5} & -\left(\begin{array}{c}
k_{6}+k_{7}+ \\
k_{13}+k_{16}
\end{array}\right) & k_{8} \\
k_{10} & k_{18} & k_{12} & k_{7} & -\left(\begin{array}{l}
k_{8}+k_{9}+ \\
k_{11}+k_{17}
\end{array}\right)
\end{array}\right)
$$

Fig. 3 The kinetic model of the pentangle reaction

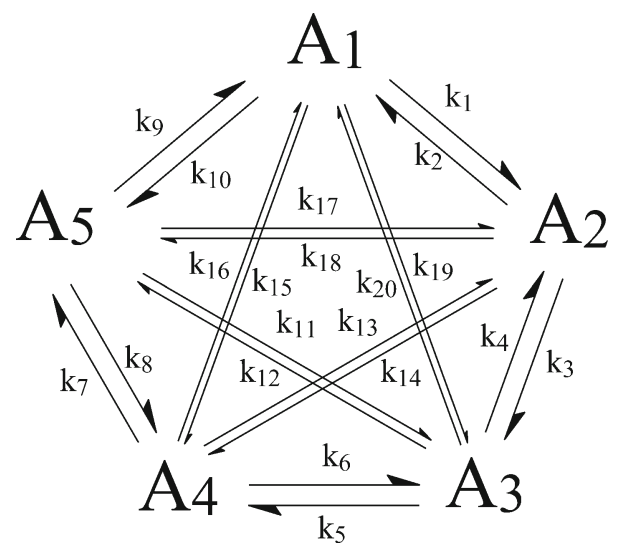


The following expressions can be obtained according to Eq. (13) for the coefficients of the characteristic polynomial of matrix $\mathbf{F}$ in Eq. (44):

$$
\begin{aligned}
& a_{6}=(-1)^{5}=-1 \\
& a_{5}=-a_{6} \operatorname{tr}(\mathbf{F}) \\
& a_{4}=-\frac{1}{2}\left(a_{5} \operatorname{tr}(\mathbf{F})+a_{6} \operatorname{tr}\left(\mathbf{F}^{2}\right)\right) \\
& a_{3}=-\frac{1}{3}\left(a_{4} \operatorname{tr}(\mathbf{F})+a_{5} \operatorname{tr}\left(\mathbf{F}^{2}\right)+a_{6} \operatorname{tr}\left(\mathbf{F}^{3}\right)\right) \\
& a_{2}=-\frac{1}{4}\left(a_{3} \operatorname{tr}(\mathbf{F})+a_{4} \operatorname{tr}\left(\mathbf{F}^{2}\right)+a_{5} \operatorname{tr}\left(\mathbf{F}^{3}\right)+a_{6} \operatorname{tr}\left(\mathbf{F}^{4}\right)\right) \\
& a_{1}=0
\end{aligned}
$$

where

$$
\begin{aligned}
\operatorname{tr}(\mathbf{F}) & =\sum_{i=1}^{5} f_{i i} \\
\operatorname{tr}\left(\mathbf{F}^{2}\right) & =\sum_{i=1}^{5} \sum_{j=1}^{5} f_{i j} f_{j i} \\
\operatorname{tr}\left(\mathbf{F}^{3}\right) & =\sum_{i=1}^{5} \sum_{j=1}^{5} \sum_{k=1}^{5} f_{i k} f_{k j} f_{j i} \\
\operatorname{tr}\left(\mathbf{F}^{4}\right) & =\sum_{i=1}^{5} \sum_{j=1}^{5} \sum_{k=1}^{5} \sum_{l=1}^{5} f_{i k} f_{k l} f_{l j} f_{j i} .
\end{aligned}
$$

To get the eigenvalues of the matrix $\mathbf{F}$, we should solve the following quartic equation $\left(\lambda_{5}=0\right)$ :

$$
a_{6} \lambda^{4}+a_{5} \lambda^{3}+a_{4} \lambda^{2}+a_{3} \lambda+a_{2}=0
$$

Equation (47) can be solved via Ferrari's formula [22]. Assuming that the eigenvalues are all distinct, the following time propagation vector is obtained:

$$
\mathbf{E}(t)=\left\{e^{\lambda_{1} t}, e^{\lambda_{2} t}, e^{\lambda_{3} t}, e^{\lambda_{4} t}, 1\right\}^{T}
$$

The columns of the matrix $\mathbf{V}$ are as follows:

$$
\begin{aligned}
& \mathbf{E}(0)=\left\{e^{\lambda_{1} t}, e^{\lambda_{2} t}, e^{\lambda_{3} t}, e^{\lambda_{4} t}, 1\right\}_{t=0}^{T}=\{1,1,1,1,1\}^{T} \\
& \dot{\mathbf{E}}(0)=\left\{\lambda_{1} e^{\lambda_{1} t}, \lambda_{2} e^{\lambda_{2} t}, \lambda_{3} e^{\lambda_{3} t}, \lambda_{4} e^{\lambda_{4} t}, 0\right\}_{t=0}^{T}=\left\{\lambda_{1}, \lambda_{2}, \lambda_{3}, \lambda_{4}, 0\right\}^{T} \\
& \ddot{\mathbf{E}}(0)=\left\{\lambda_{1}^{2} e^{\lambda_{1} t}, \lambda_{2}^{2} e^{\lambda_{2} t}, \lambda_{3}^{2} e^{\lambda_{3} t}, \lambda_{4}^{2} e^{\lambda_{4} t}, 0\right\}_{t=0}^{T}=\left\{\lambda_{1}^{2}, \lambda_{2}^{2}, \lambda_{3}^{2}, \lambda_{4}^{2}, 0\right\}^{T}
\end{aligned}
$$




$$
\begin{aligned}
\dddot{\mathbf{E}}(0) & =\left\{\lambda_{1}^{3} e^{\lambda_{1} t}, \lambda_{2}^{3} e^{\lambda_{2} t}, \lambda_{3}^{3} e^{\lambda_{3} t}, \lambda_{4}^{3} e^{\lambda_{4} t}, 0\right\}_{t=0}^{T}=\left\{\lambda_{1}^{3}, \lambda_{2}^{3}, \lambda_{3}^{3}, \lambda_{4}^{3}, 0\right\}^{T} \\
\dddot{\mathbf{E}}(0) & =\left\{\lambda_{1}^{4} e^{\lambda_{1} t}, \lambda_{2}^{4} e^{\lambda_{2} t}, \lambda_{3}^{4} e^{\lambda_{3} t}, \lambda_{4}^{4} e^{\lambda_{4} t}, 0\right\}_{t=0}^{T}=\left\{\lambda_{1}^{4}, \lambda_{2}^{4}, \lambda_{3}^{4}, \lambda_{4}^{4}, 0\right\}^{T}
\end{aligned}
$$

With the help of Eq. (49), the matrix $\mathbf{V}$ and its inverse $\mathbf{V}^{-1}$ can easily be constructed:

$$
\mathbf{V}=\left(\begin{array}{ccccc}
1 & \lambda_{1} & \lambda_{1}^{2} & \lambda_{1}^{3} & \lambda_{1}^{4} \\
1 & \lambda_{2} & \lambda_{2}^{2} & \lambda_{2}^{3} & \lambda_{2}^{4} \\
1 & \lambda_{3} & \lambda_{3}^{2} & \lambda_{3}^{3} & \lambda_{3}^{4} \\
1 & \lambda_{4} & \lambda_{4}^{2} & \lambda_{4}^{3} & \lambda_{4}^{4} \\
1 & 0 & 0 & 0 & 0
\end{array}\right)
$$

Finally, only the elements of matrix $\mathbf{F}_{\mathbf{C}_{0}}$ should be generated:

$$
\begin{aligned}
& \left(\mathbf{F}_{\mathbf{C}_{0}}\right)_{p 1}=c_{0 p} \\
& \left(\mathbf{F}_{\mathbf{C}_{0}}\right)_{p 2}=\sum_{q=1}^{5} f_{p q} c_{0 q} \\
& \left(\mathbf{F}_{\mathbf{C}_{0}}\right)_{p 3}=\sum_{q=1}^{5} f_{p q}\left(\mathbf{F}_{\mathbf{C}_{0}}\right)_{q 2}=\sum_{q=1}^{5} \sum_{u=1}^{5} f_{p q} f_{q u} c_{0 u} \\
& \left(\mathbf{F}_{\mathbf{C}_{0}}\right)_{p 4}=\sum_{q=1}^{5} f_{p q}\left(\mathbf{F}_{\mathbf{C}_{0}}\right)_{q 3}=\sum_{q=1}^{5} \sum_{u=1}^{5} \sum_{v=1}^{5} f_{p q} f_{q u} f_{u v} c_{0 v} \\
& \left(\mathbf{F}_{\mathbf{C}_{0}}\right)_{p 5}=\sum_{q=1}^{5} f_{p q}\left(\mathbf{F}_{\mathbf{C}_{0}}\right)_{q 4}=\sum_{q=1}^{5} \sum_{u=1}^{5} \sum_{v=1}^{5} \sum_{w=1}^{5} f_{p q} f_{q u} f_{u v} f_{v w} c_{0 w}
\end{aligned}
$$

where $p=1,2,3,4,5$. The concentrations of the components can now be obtained analytically:

$$
c_{j}=\sum_{k=1}^{5} \sum_{l=1}^{5}\left(\mathbf{F}_{\mathbf{C}_{0}}\right)_{j k}\left(\mathbf{V}^{-1}\right)_{k l} e^{\lambda_{l} t} \quad(j=1,2,3,4,5)
$$

Degenerate eigenvalues can be handled as before.

\section{Simulations for the quadrangle and pentangle reactions}

First, we make a kinetic simulation for the quadrangle reaction with the following parameters: $c_{0 p}=0.1 p$ mol dm${ }^{-3}(p=1,2,3,4)$ and $k_{q}=q \mathrm{~h}^{-1}(q=1,2, \ldots, 12)$. The coefficient matrix of the reaction system: 
Fig. 4 A kinetic simulation for the quadrangle reaction (see the text for details)

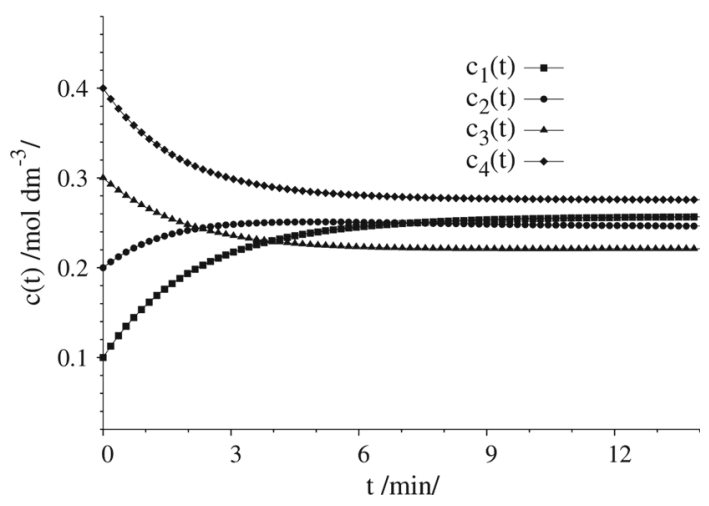

$$
\mathbf{F}=\left(\begin{array}{cccc}
-18 & 2 & 10 & 7 \\
1 & -17 & 4 & 11 \\
9 & 3 & -20 & 5 \\
8 & 12 & 6 & -23
\end{array}\right)
$$

The cubic equation obtained for the eigenvalues of the $\mathbf{F}$ matrix is as follows $\left(\lambda_{4}=0\right)$ :

$$
\lambda^{3}+78 \lambda^{2}+1949 \lambda+15364=0
$$

With the help of Cardano's formula, the following eigenvalues can be obtained:

$$
\begin{aligned}
& \lambda_{1} \approx-16.24326197410 \mathrm{~h}^{-1} \\
& \lambda_{2} \approx-33.63600338329 \mathrm{~h}^{-1} \\
& \lambda_{3} \approx-28.12073464261 \mathrm{~h}^{-1}
\end{aligned}
$$

The approximate $\mathbf{V}$ matrix can now be constructed according to Eq. (39):

$$
\mathbf{V} \approx\left(\begin{array}{cccc}
1 & -16.24326 & 263.84356 & -4285.68006 \\
1 & -33.63600 & 1131.38072 & -38055.12585 \\
1 & -28.12073 & 790.77572 & -22237.19410 \\
1 & 0 & 0 & 0
\end{array}\right)
$$

The exact $\mathbf{F}_{\mathbf{C}_{0}}$ matrix is as follows:

$$
\mathbf{F}_{\mathbf{C}_{0}}=\left(\begin{array}{cccc}
0.1 & 4.4 & -129 & 3906 \\
0.2 & -2.3 & -90.9 & 3306.7 \\
0.3 & -2.5 & 75.5 & -2221.7 \\
0.4 & -4.2 & 144.4 & -4991
\end{array}\right)
$$

Now we can draw the concentration profiles of the chemical components based on Eq. (42) (see Fig. 4). 
Fig. 5 A kinetic simulation for the pentangle reaction (see the text for details)

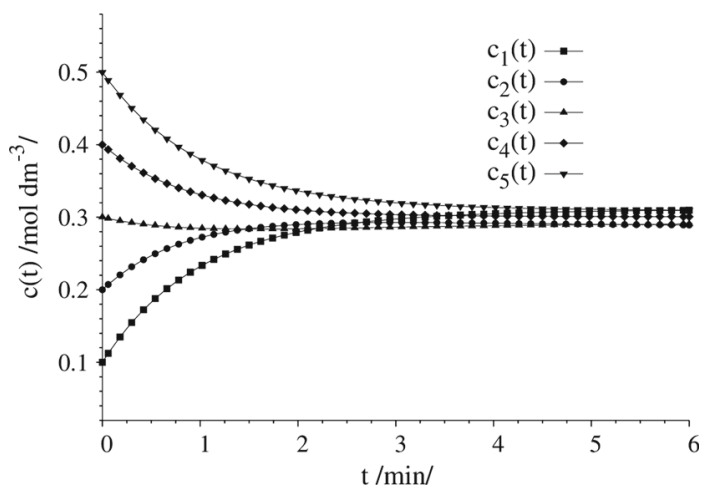

Next, let us make a kinetic simulation for the pentangle reaction with the following parameters: $c_{0 p}=0.1 p \mathrm{~mol} \mathrm{dm}^{-3}(p=1,2,3,4,5)$ and $k_{q}=q \mathrm{~h}^{-1}(q=1,2$, $\ldots, 20)$. The coefficient matrix of the reaction system:

$$
\mathbf{F}=\left(\begin{array}{ccccc}
-45 & 2 & 20 & 16 & 9 \\
1 & -37 & 4 & 13 & 17 \\
19 & 3 & -41 & 6 & 11 \\
15 & 14 & 5 & -42 & 8 \\
10 & 18 & 12 & 7 & -45
\end{array}\right)
$$

The quartic equation for the eigenvalues of the $\mathbf{F}$ matrix $\left(\lambda_{5}=0\right)$ :

$$
\lambda^{4}+210 \lambda^{3}+16188 \lambda^{2}+541169 \lambda+6597831=0
$$

Equation (59) can be solved with the help of Ferrari's formula:

$$
\begin{aligned}
& \lambda_{1} \approx-67.48255066579 \mathrm{~h}^{-1} \\
& \lambda_{2} \approx-61.77496364344 \mathrm{~h}^{-1} \\
& \lambda_{3} \approx-33.50521325294 \mathrm{~h}^{-1} \\
& \lambda_{4} \approx-47.23727243783 \mathrm{~h}^{-1}
\end{aligned}
$$

The approximate $\mathbf{V}$ matrix can now be constructed based on Eq. (50):

$$
\mathbf{V} \approx\left(\begin{array}{ccccc}
1 & -67.48255 & 4553.89464 & -307308.42606 & 20737956.43193 \\
1 & -61.77496 & 3816.14613 & -235742.28863 & 14562971.30955 \\
1 & -33.50521 & 1122.59932 & -37612.92945 & 1260229.22232 \\
1 & -47.23727 & 2231.35991 & -105403.35585 & 4978967.03620 \\
1 & 0 & 0 & 0 & 0
\end{array}\right)
$$


The exact $\mathbf{F}_{\mathbf{C}_{0}}$ matrix is as follows:

$$
\mathbf{F}_{\mathbf{C}_{0}}=\left(\begin{array}{ccccc}
0.1 & 12.8 & -814.3 & 53275.4 & -3539502.4 \\
0.2 & 7.6 & -562.5 & 39160 & -2653795.1 \\
0.3 & -1.9 & 175.4 & -13589.7 & 990319.1 \\
0.4 & -7 & 490.9 & -34146.3 & 2355971.9 \\
0.5 & -11.5 & 710.5 & -44699.4 & 2847006.5
\end{array}\right)
$$

The concentration profiles of the chemical components based on Eq. (52) can be seen in Fig. 5.

\section{Conclusions}

This paper presents a simple algebraic model to handle the kinetic problems of firstorder reaction networks. In order to solve the kinetic problems within the model, only the eigenvalues of the coefficient matrix of the reaction system should be determined. For the triangle, quadrangle and pentangle reactions $(K \leq 5)$, analytical solutions do exist. The application of the model for larger systems $(K>5)$ is straightforward, however, the eigenvalues should be computed numerically.

Acknowledgments The authors thank the TÁMOP Project (TÁMOP-4.2.2.A-11/1/KONV-2012-0047) for financial support.

\section{References}

1. J. Wei, C.D. Prater, Adv. Catal. 13, 203 (1962)

2. D.M. Himmelblau, C.R. Jones, K.B. Bischoff, Ind. Eng. Chem. Fundam. 6, 539 (1967)

3. L. Pogliani, M.N. Berberan-Santos, J.M.G. Martinho, J. Math. Chem. 20, 193 (1996)

4. C. Chu, Chem. Eng. Sci. 26, 1651 (1971)

5. J. Chrastil, Comput. Chem. 17, 103 (1993)

6. V.I. Korobov, V.F. Ochkov, Chemical Kinetics with Mathcad and Maple (Springer, Wien, 2011)

7. M.N. Berberan-Santos, L. Pogliani, J.M.G. Martinho, React. Kinet. Catal. Lett. 54, 287 (1995)

8. G.R. Eykholt, Water Res. 33, 814 (1999)

9. M. Socol, I. Baldea, J. Math. Chem. 45, 478 (2009)

10. N.A.S.B. Hasan, P. Balasubramanian, Ind. Eng. Chem. Res. 52, 10594 (2013)

11. M. Socol, I. Baldea, J. Chin. Chem. Soc. 53, 773 (2006)

12. S. Karmakar, B. Mandal, J. Phys. Chem. A 118, 7672 (2014)

13. U. Luther, K. Rost, Electron. Trans. Numer. Anal. 18, 91 (2004)

14. V. Chellaboina, S. Bhat, M.M. Haddad, D.S. Bernstein, IEEE Control Syst. 29, 60 (2009)

15. G. Schmitz, J. Chem. Educ. 82, 1091 (2005)

16. S. Le Vent, J. Chem. Educ. 80, 89 (2003)

17. P. Waage, C.M. Gulberg, J. Chem. Educ. 63, 1044 (1986)

18. B.R. Ramachandran, A.M. Halpern, J. Chem. Educ. 73, 686 (1996)

19. D. Kalman, Math. Mag. 73, 313 (2000)

20. H.K. Krishnapriyan, J. Chem. Inf. Comput. Sci. 35, 196 (1995)

21. A. Fathi, N. Sharifan, Appl. Comput. Math. 2, 24 (2013)

22. M. Falati, G. Hojjati, J. Math. Chem. 49, 2210 (2011) 